\title{
Designing of a novel multisubunit vaccine against Nipah virus structural proteins: A reverse vaccinology approach
}

khalid Mohamed Adam ( $\sim$ dr.aboalbasher@gmail.com )

College of Applied Medical Science, University of Bisha https://orcid.org/0000-0003-2381-1968

\section{Research Article}

Keywords: Multisubunit, Vaccine, Nipah virus, Immunoinformatics, Multi-epitope

Posted Date: September 28th, 2021

DOI: https://doi.org/10.21203/rs.3.rs-935912/v1

License: @ (i) This work is licensed under a Creative Commons Attribution 4.0 International License. Read Full License 


\section{Abstract \\ Background}

The significant public health risk posed by NiV zoonosis and the lack of effective countermeasures against the intermittent outbreaks of the disease in the South and Southeast Asia region have entailed an imperative search for a protective vaccine to prevent or mitigate its epidemic potentiality. This is an endeavor to design an effective, safe multisubunit vaccine using an in silico reverse vaccinology approach.

\section{Methods}

The epitopes used for the construction of the candidate vaccine were meticulously predicted from five viral structural proteins (G, F, M, N, P) using several immunoinformatics tools to assess different epitope characteristics, namely, VaxiJen server for antigenicity, IEDB immunogenicity tool for immunogenicity, AlgPred server for allergenicity, ToxinPred for toxigenicity, IFNepitope server for interferon-gamma induction, Protparam server for physicochemical properties, GROMACS for simulation and simulation dynamics analysis, and finally, SnapGene tool for molecular cloning.

\section{Results}

The proposed vaccine molecule consisted of 501 amino acids, encompassing 7 B cell epitopes, 14 CTL epitopes, and 4 HTL epitopes. The physiochemical parameters of the vaccine construct showed a molecular weight of $54.6 \mathrm{kDa}$, an acidic stable molecule with an instability index of 38.3 , aliphatic index of 62.89 , and grand average of hydropathicity of -0.476 . Moreover, the docking results and simulation dynamics of the vaccine molecule and TLR-3 showed global energy of $1.58 \mathrm{Kcal} / \mathrm{mol}$, atomic contact energy of $2.98 \mathrm{Kcal} / \mathrm{mol}$, and RMSD of $0.65 \mathrm{~nm}$. The radius gyration showed a relatively steady value throughout the simulation period. a suggestive result of a stable compact structure and a promisingly effective vaccine construct.

\section{Conclusion}

In summary, the overall results of the multi-subunit vaccine molecule are suggestive of a promisingly effective vaccine against NiV infection in humans with a relatively stable compact structure, however, further experimental validation and assessment of pathogenic priming and autoimmunity induction are recommended.

\section{Introduction}

In September 1998 an outbreak of a viral origin occurred in Malaysia and Singapore, a cause of which was later isolated, identified, and named Nipah virus (NiV) after a Malaysian village in which it was first discovered [1]. Since then, similar NiV outbreaks occur intermittently in the South and South-East Asia region, threatening of grave health and economic repercussions and a full-blown global pandemic [2,3]. NiV zoonosis occurs in both animals and humans, and the primary reservoir identified to be the fruit bat. Infection in humans ranges from subclinical to acute respiratory distress and fatal febrile encephalitis, with case fatality rates ranging from $40-100 \%[3,4]$. The clinical presentations of the disease include severe influenza-like symptoms such as fever, myalgia, and headache. Encephalitis symptoms appear within a week of infection followed by rapid deterioration into coma and death within few days [5]. Infection can be non-encephalitic, and cases of relapse or late-onset encephalitis have been reported [6, 7].

NiV, a paramyxovirus, comprises, along with Hendra virus $(\mathrm{HeV})$ and Cedar virus, the genus Henipavirus. The fruit bats of the genus Pteropus have been identified as infection reservoirs of NiV $[8,9]$, and humans can get the infection through either food-borne route from food contaminated with saliva, excrement, or semen of carrier bats, or through direct contact with infected humans and animals $[10,11,4]$. The viral infection is mediated by the highly conserved Ephrin-B2/B3 receptors [12], which are mainly expressed in the brain, endothelial, and smooth muscle cells in the arterial vessels [13]. NiV is a single-stranded negative-sense RNA enveloped virus with a genome size of $18.2 \mathrm{~kb}$, which encompasses 6 viral genes encoding 9 viral proteins: nucleoprotein $\mathrm{N}$, phosphoprotein $\mathrm{P}$, nonstructural proteins $\mathrm{W}, \mathrm{V}$, and $\mathrm{C}$, matrix protein $\mathrm{M}$, fusion protein F, glycoprotein $\mathrm{G}$, and large polymerase $\mathrm{L}$ [14]. The binding to the host Ephrin receptors is mediated by the viral glycoprotein $\mathrm{G}$, while the membrane fusion and viral entry is the function of protein $\mathrm{F}$ [14-16]. The proteins $\mathrm{N}$ and $\mathrm{P}$ form a complex that binds the viral RNA forming the nucleocapsid protein that coats the viral RNA [17]. The viral genetic material is replicated with the help of the RNA polymerase L, furthermore, protein M mediates the budding and release of viral particles from the host cells [18]. On the other hand, the nonstructural accessory proteins W, V, and C are key components for innate immune response evasion [19, 10]. The World Health Organization (WHO) has prioritized NiV infection for research and development in emergency contexts by listing it among diseases pose the greatest public health risk due to their epidemic potential [10], a fact aggravated by the lack of approved protective vaccines and/or effective treatment, which entailed the search for an efficient, safe prophylactic agent against the zoonotic infection. Few attempts were made towards the development of human vaccines, some were glycoprotein G-based subunit vaccines, and others were vector-based recombinant vaccines of proteins $F$ and $G$. These vaccines showed promising results in various animal models, however, not proved protective in humans $[21,14,5,22]$. Therefore, the aim of the present study is to exploit the immunoinformatics tools and the viral structural proteins data to design a multi-epitope vaccine capable of eliciting a protective humoral and cell-mediated immune response against NiV. Two major genetic lineages of NiV were identified thus far, namely, NiV Malaysia, and NiV Bangladesh, share $92 \%$ of nucleotide homology and most of the variations reside in the region of non-structural genes $[23,24,4]$, therefore, here, the construction of the candidate vaccine was based entirely on the viral structural proteins.

\section{Materials And Methods}




\section{Retrieval of viral protein sequences}

The amino acid sequences of 5 structural viral proteins; glycoprotein $\mathrm{G}$ of 602 amino acids (accession No. Q9IH62), fusion protein $\mathrm{F}$ of 546 amino acids (accession No. Q9IH63.1), matrix protein M of 352 amino acids (accession No. Q9IK90.1), nucleoprotein N of 532 amino acids (accession No. Q9IK92.1), and phosphoprotein P of 709 amino acids (accession No. Q9IK91.1) were retrieved from NCBI protein database (https://www.ncbi.nlm.nih.gov/ protein) in FASTA format.

\section{Antigenicity prediction}

The retrieved amino acid sequences of all 5 proteins were screened for antigenicity with Vaxijen 2.0 server, an alignment-independent prediction tool of protective antigen, and the threshold value for antigenicity score was 0.4 (http://www.ddg-pharmfac.net/vaxijen/VaxiJen/VaxiJen.html) [25].

\section{Cytotoxic T lymphocyte (CTL) epitopes prediction}

The CTL epitopes from all proteins with antigenicity score more than 0.4 were predicted using the artificial neural network algorithm-based NetCTL 1.2 server (http://www.cbs.dtu.dk/services/NetCTL/). The process is based on MHC-I binding peptide prediction, proteasomal C-terminal cleavage, and transportation efficiency of transporter-associated antigen processing (ATP). The threshold value was set to 0.75 , which indicates $80 \%$ sensitivity and $97 \%$ specificity [26]. The predicted CTL epitopes were then screened independently for antigenicity using Vaxijen 2.0 server. This is followed by scanning the antigenic peptides for toxicity using the ToxinPred server, with a threshold value of 0.0 (https://webs.iiitd.edu.in/raghava/toxinpred/multi_submit.php) [27]. The nontoxic epitopes were then subjected to immunogenicity screening using the class I immunogenicity tool of Immune Epitope Database (IEDB) version 2.22 (http://tools.iedb.org/immunogenicity/) [28].

\section{Helper T lymphocyte (HTL) epitopes prediction}

The MHC-II binding tool of IEDB (http://tools.iedb.org/mhcii/) was used to predict the HTL epitopes for 7-allele HLA reference set, HLA-DRB1*03:01, HLADRB1*07:01, HLA-DRB1*15:01, HLA-DRB3*01:01, HLA-DRB3*02:02, HLA-DRB4*01:01, HLA-DRB5*01:01. The predicted peptide binding affinity is expressed in units of IC50 nM, the lower the number the higher the affinity, therefore, peptides with IC50 values $<50 \mathrm{nM}$ are considered of high binding affinity, $<500 \mathrm{nM}$ intermediate affinity, and $<5000 \mathrm{nM}$ low affinity. For each peptide, a percentile rank is computed by comparing the peptide's score against the scores of five million random 15 mers peptides selected from the SWISSPROT database. The lower the percentile rank the higher the affinity [29].

The resultant epitopes with low percentile ranks were then screened for allergenicity with AlgPred server (https://webs.iiitd.edu.in/raghava/ algpred/submission.html), using the support vector machine (SVM) module-based on the amino acid composition as the prediction approach [30]. The nonallergenic epitopes were then screened for antigenicity and toxicity status with VaxiJen 2.0 server and ToxinPred server, respectively. To explore the ability of the resultant epitopes to induce the production of Interferon-gamma (IFN- $\gamma$ ) by MHC-II activated CD4 + T helper cells, IFNepitope server (http://crdd.osdd.net/raghava/ifnepitope/) was used following the Motif and SVM hybrid approach [31]. The predicted epitopes were then manually inspected for overlapping.

\section{Population coverage}

The worldwide population coverage of the predicted epitopes for MHC-I and MHC-II alleles was conducted using the population coverage tool of IEDB (http://tools.iedb.org/population/) [32]. The population coverage was calculated for class I and class II independently and combined. The assessed MHC-I alleles were HLA-B*15:01, HLA-A*30:02, HLA-A*01:01, HLA-B*40:01, HLA-B*07: 02, HLA-B*51:01, HLA-A*68:02, HLA-A*02:01, HLA- $A^{\star} 02: 06, H L A-B * 08: 01, H L A-$ $A * 02: 03, H L A-A * 33: 01$, HLA-A*24:02, HLA-A*23:01, HLA-B*44:03, HLA-B*44: 02, HLA-A*31:01, HLA-B*53:01, HLA-A*11:01, HLA- A*68:01, HLA-A*30:01, HLA$B \star 57: 01, H L A-A * 03: 01, H L A-A * 26: 01, H L A-B \star 58: 01, H L A-A * 32: 01, H L A-B \star 35: 0$, and for MCH-II the alleles assessed were HLA-DRB1*07:01, HLA-DRB1*15:01, HLA-DRB3*01:01.

\section{B-cell epitope and antibody prediction}

The B cell epitopes are key players in humoral immunity, they bind surface immunoglobulins and derive the B cells into the immune response [33]. The antibody epitope prediction tool of the Immune Epitope Database and Analysis Resource (IEDB) (http://tools.iedb.org/bcell/) was used to predict the linear B cell epitopes with BepiPred linear epitope prediction method [34], Emini surface accessibility prediction method [35], and Kolaskar and Tongaonkar antigenicity method [36]. The epitopes were selected for inclusion in the proposed vaccine construct only if they were predicted by the former prediction method and fulfilled the surface accessibility and antigenicity of the latter two methods. The class of antibodies induced by these epitopes was predicted using the lgPred server (https://webs.iiitd.edu.in/raghava/igpred/) by scanning antibody-specific motifs in the peptide sequence [37].

\section{Multiepitope vaccine construction}

For devising the final vaccine construct, the predicted CTL, HTL, and B cell epitopes were joined together using linkers to ensure efficient vaccine construction and proper subunit separation. The B cell epitopes were linked together and with CTL epitopes with AAY linker, while HTL epitopes were linked together and with CTL epitopes with GPGPG linker. At the N-terminal, a cysteine residue was added to facilitate the future conjugation of the vaccine construct with the carrier protein [38]. Furthermore, to ensure efficient purification of the proposed vaccine, a four amino acid (EPEA) tag was added at the C-terminal of the construct [39]. To explore any potential autoimmunity, BLASTp of the candidate vaccine against the human proteome in the Uniprot database was conducted to screen for considerable similarity with human proteins indicated by $>40 \%$ identity and E-value [40]. The final construct was also assessed for antigenicity and allergenicity using VaxiJen 2.0 server and AlgPred server, respectively. The ExPASy ProtParam tool (https://web.expasy.org/protparam/) was used to determine the physicochemical properties, including hydropathicity, charge, instability, half-life, the value of theoretical isoelectric point, and molecular weight [41]. 


\section{Structure modeling and validation}

Initially, the secondary structure of the candidate vaccine was determined using the PSIPRED server (http://bioinf.cs.ucl.ac.uk/psipred/) [42]. The candidate vaccine 3D structure homology-modeling was carried out using the ExPASy SWISS-MODEL tool. The resultant structure was further analyzed and validated with the ProSA-web server (https://prosa.services.came.sbg.ac.at/prosa.php) [43], Ramachandran plot analysis using MolProbity server (http://molprobity.biochem.duke.edu/logout_destroy.php) [44], and ERRAT server (https://servicesn.mbi.ucla.edu/ERRAT/) [45].

\section{Molecular docking and simulation}

The binding and interaction of the antigenic molecules with a specific immune receptor is a key step for proper efficacious immune response, therefore, it is essential to examine the interaction between the resultant vaccine and a class of pattern recognition receptor that is responsible for the initiation of the innate immune response. The vaccine-Toll-like receptor 3 docking was carried out using the PatchDock server (https://bioinfo3d.cs.tau.ac.il/PatchDock/) [46], and HADDOCK server 2.4 (https://wenmr.science.uu.nl/haddock2.4/) [47] using servers' default parameters.

The docked complex was then subjected to pre-simulation modification using protein prep wizard [48] and PyMOL software. The molecular simulation and molecular dynamics analysis were carried out using Groningen Machine for Chemical Simulations (GROMACS) molecular dynamics package, using the CHARMM TIP3P force field, and TIP4P-FQ water model. The calibration and charge neutralization of the simulation system were carried out. The system temperature and pressure were equilibrated at $300 \mathrm{~K}$ and 1 bar for a $100 \mathrm{~ns}$ equilibration period with an interval of $2 \mathrm{fs}$. The simulation process was visualized using the VMD tool. The root mean square deviation (RMSD) and radius of gyration analyses were determined.

\section{Immune response simulation}

The immune response to the proposed vaccine was carried out using C-ImmSim server 10.1 (http://www.cbs.dtu.dk/services/C-ImmSim-10.1/) [49], which is an agent-based tool that uses a position-specific scoring matrix (PSSM) and machine learning for the prediction of immune responses. It simulates the production of lymphoid and myeloid cells by hematopoietic stem cells in the bone marrow and the immune response in the thymus and lymph node. The simulation parameters used were random seed: 12,345 , simulation steps: 100 , and simulation volume: $10 \mu \mathrm{L}$. The default injection schedule with the antigen name, injection time: 0, and injection amount: 1000 .

\section{In silico molecular cloning}

The amino acid sequence of the vaccine construct was subjected to reverse translation and codon optimization with the JAVA codon adaptation tool (Jcat) (http://www.jcat.de) [50]. The GC content of the resultant DNA sequence is $52 \%$ suggesting efficient translation and transcription. The sequence was then used for in silico molecular cloning using expression plasmid vector pET28 (+) of E. coli (strain K12) [51], and Ncol and Notl restriction sites were introduced the $\mathrm{N}$ and C-terminals of the construct, respectively. The molecular cloning was carried out using SnapGene tool version 5.2.

\section{Results}

\section{T cell epitopes prediction}

The antigenicity screening of the retrieved NiV protein sequences showed a score greater than the threshold value of 0.4 for glycoprotein $\mathrm{G}$ (0.5095), fusion protein $\mathrm{F}(0.5395)$, nucleoprotein $\mathrm{N}(0.5594)$, and phosphoprotein $\mathrm{P}(0.5767)$, suggesting probable antigenicity, while the antigenicity score of the matrix protein $\mathrm{M}(0.3969)$ was indicative of non-antigenic nature of the protein, therefore it was excluded from further analysis. The 4 antigenic proteins were then submitted to the NetCTL server for CTL epitope prediction, resulting in 12 potential epitopes from protein G, out of which 11 were nontoxic and showed a positive immunogenicity score, all were included in the final vaccine construct. For protein F, $21 \mathrm{CTL}$ potential epitopes were predicted, of which 4 were nontoxic, of these, 2 with a positive immunogenicity score were included in the vaccine construct. Eight CTL potential epitopes were predicted from the nucleoprotein $\mathrm{N}$, all were nontoxic, and the 2 included in the final vaccine construct were the only ones with a positive immunogenicity score. For phosphoprotein P, 10 CTL potential epitopes were predicted, 5 of which were nontoxic and only 3 epitopes were selected for the final vaccine construct for their positive immunogenicity score. All the ultimately selected epitopes for inclusion in the vaccine construct showed antigenicity scores greater than the threshold value (Table 1)

Table 1: Characteristics of predicted CTL epitopes 


\begin{tabular}{lllllll}
\hline Protein & Peptide & Prediction score & $\begin{array}{l}\text { VaxJen } \\
\text { Score }\end{array}$ & SVM score & Toxicity & Immunogenicity score \\
\hline $\mathbf{G}$ & STDNQAVIK & 1.4497 & 0.4021 & -1.07 & Non-toxin & 0.03732 \\
& STASINENV & 0.9814 & 0.5714 & -1.01 & Non-toxin & 0.05046 \\
& AMDEGYFAY & 3.007 & 0.6169 & -0.83 & Non-toxin & 0.25913 \\
& CSAVYNNEF & 1.8928 & 0.4829 & -0.85 & Non-toxin & 0.09759 \\
& SAVYNNEFY & 1.4353 & 0.5888 & -1.23 & Non-toxin & 0.17688 \\
& AVYNNEFYY & 0.7584 & 0.6013 & -1.21 & Non-toxin & 0.17688 \\
& DSLGQPVFY & 1.0398 & 0.621 & -1.31 & Non-toxin & 0.0105 \\
$\mathbf{F}$ & ECSVGILHY & 0.9645 & 0.4284 & -0.44 & Non-toxin & 0.15566 \\
& QITAGVALY & 1.2334 & 0.4052 & -1.22 & Non-toxin & 0.15037 \\
$\mathbf{N}$ & QTKFAPGGY & 1.3023 & 1.0211 & -0.99 & Non-toxin & 0.12386 \\
& SSDQVAELA & 1.2561 & 0.6207 & -1.09 & Non-toxin & 0.04569 \\
$\mathbf{P}$ & QLDPVVTDV & 0.8144 & 0.6201 & -1.24 & Non-toxin & 0.12082 \\
& ETDLVHLEN & 0.9896 & 0.5078 & -0.82 & Non-toxin & 0.11583 \\
& YHADHLGDY & 0.763 & 0.4871 & -0.61 & Non-toxin & 0.09764 \\
\hline
\end{tabular}

Percentile rank less than 10 was used as the cut-off value for the selection of predicted HTL epitopes which resulted in 36 peptides from protein G, one of which was selected for inclusion in the final construct, despite 5 were non-allergenic, 3 were nontoxic and 2 were antigenic, but only one showed IFNinducibility. For protein F, none of the 18 predicted HTL epitopes showed IFN- inducibility, while 2 epitopes were selected from the 20 predicted HTL epitopes from nucleoprotein $\mathrm{N}$, and one epitope from protein $\mathrm{P}$, that fulfilled the selection criteria (Table 2).

Table 2: Characteristics of predicted HTL epitopes

\begin{tabular}{llllll}
\hline Protein & Peptide & Percentile rank & Toxicity & Antigenicity score & IFN-gamma \\
\hline $\mathbf{G}$ & LGQPVFYQASFSWDT & 9.8 & Non-toxin & 0.8008 & Positive \\
$\mathbf{N}$ & GKTPFVDSRAYGLRI & 1.4 & Non-toxin & 0.4445 & Positive \\
& QSDLNTIKSLMLLYR & 0.64 & Non-toxin & 0.5368 & Positive \\
$\mathbf{P}$ & VIKTLIRTHIKDREL & 9.5 & Non-toxin & 0.6214 & Positive \\
\hline
\end{tabular}

\section{Population coverage}

The analysis of world population coverage of the selected epitopes for MHC-I and MHC-II alleles. The coverage of HLA class I restricted epitopes was $98.59 \%$ of the world population, and the coverage of HLA class II restricted epitopes was $69.81 \%$ of the world population, while the coverage of the combined HLA class I and class II was $99.57 \%$ of the world population, this suggests a remarkably high population coverage of the given multisubunit vaccine (Fig. 1 ).

\section{B cell epitope prediction}

The B cell epitopes derive their importance from being key players in the adaptive humoral immune response. Upon their recognition by B lymphocytes, they trigger antibody production which is an important defense process against viral infection. Three methods were employed to ensure proper epitopes prediction, epitopes predicted by the Bepipred prediction method were assessed for surface accessibility, flexibility, and antigenicity by Emini and Kolaskar \& Tongaonkar methods, respectively. Only epitopes predicted by the three methods were included in the final vaccine construct. Ultimately, 7 epitopes of variable sequence length were predicted by the three methods, one from proteins $\mathrm{G}, \mathrm{F}, \mathrm{M}$, and P, each, and three from protein N. All predicted epitopes induce lgG antibodies.

\section{Multi-subunit vaccine construction}

The final vaccine construct comprised of 7 B cell epitopes, 14 CTL epitopes, 4 HTL epitopes, 2 linkers, EPEA tag at the C-terminal, and a cysteine residue, resulting in a sequence of 501 amino acids:

\section{CAAYVRFENTTSDKGKIPSKVIKSYYGTMDIKKINEGLLDAAYNTYSRLEDRRVRPTSSGDLYYAAYIKSISSESMEGVSDFSPSSWEHGGYLDKVEPEIDENGAAYKTARDSSH}

\section{Physiochemical properties of the vaccine construct}

The properties of the novel vaccine assessed by the ProtParam server showed a molecular weight of $54.6 \mathrm{KDa}$ and a theoretical isoelectric point (PI) of 4.78, suggesting an acidic nature, a total of 61 negatively charged residues and 41 positively charged residues, which make it a readily soluble protein in blood pH. 
The estimated half-life is 1.2 hours in mammalian reticulocytes in vitro, $>20$ hours in yeast in vivo, and $>10$ hours in $E$. coli in vivo. The instability index was computed to be 38.30 , which classifies the protein as a stable molecule. The vaccine construct also showed an aliphatic index of 62.89 , indicating a thermostable protein. The grand average of hydropathicity (GRAVY) was -0.476 , a negative value means a hydrophilic molecule.

\section{Tertiary structure modeling and analysis}

The results of secondary structure prediction by PSIPRED sever showed predominant helix and coil structures (Fig 2), homology modeling was then carried out using the SWISS Model tool to predict the tertiary structure of the multi-subunit construct which resulted in 29 modeling templates. The top three templates with the best sequence coverage and larger identity $62.14 \%$ each, were selected for building the 3D models of the protein. The quality of these models was then assessed and validated using Ramachandran plot, ERRAT tool to calculate the overall quality factor, VERIFY3D tool, and ProSA-web z-score based on Xray crystallography and NMR analysis. The best-predicted model showed $93.1 \%$ of the residues fall within the favorable region in the Ramachandran plot (Fig 3 ), and overall quality factor of $90.426 \%$ calculated by blotting the statistics of non-bonded interactions between different atom types and the error function value, against a position of a 9-residue sliding window, compared with statistics from a highly refined structure. The value is less than the $91 \%$ threshold for an average quality (Fig 4). The model also showed a Z-score of -3.84 determined by both x-ray crystallography and NMR analysis (Fig 5)

\section{Molecular docking and dynamics analysis}

The docking of the novel vaccine construct with Toll-like receptor 3 (PDB ID: 1ziw) was performed with the PatchDock server, the generated docking solutions were sorted based on the geometric shape complementarity score, the approximate interface area of the complex, and the atomic contact energy. The top 10 protein-receptor docking solutions were submitted to the FireDock server for interaction refinement. The selected docking solution had a globale energy of $-1.58 \mathrm{Kcal} / \mathrm{mol}$, attractive van der Waals force of $-9.13 \mathrm{Kcal} / \mathrm{mol}$, repulsive van der Waals force of $1.83, \mathrm{Kcal} / \mathrm{mol}$, and atomic contact energy of $2.98 \mathrm{Kcal} / \mathrm{mol}$ (Fig.6). The molecular dynamics simulation results showed a root mean square deviation (RMSD) of $0.65 \mathrm{~nm}$ and the radius of gyration value with a relatively minor fluctuation throughout the 1000 ps period of simulation (Fig. 7,8).

\section{Immune response simulation}

Assessing the immune response to the designed vaccine is an important step towards ensuring vaccine efficiency. The simulation results showed increased and sustained levels of memory and active B cell population. A similar result was shown by $\mathrm{T}$ cell population, the memory and active $\mathrm{T}$ helper cells maintained a high level for the entire simulation period. The immunoglobulins level, on the other hand, peaked by the second week of simulation and then regressed to relatively acceptable levels nonetheless. The concentration of cytokines and interleukins showed for more than half the simulation period followed by a gradual decline (Fig. 9), suggesting sustained humoral and cell-mediated immune response.

\section{In silico molecular cloning}

The Java codon adaptation tool was used to optimize the novel vaccine sequence prior to the insertion of the vaccine DNA sequence into the pET28 (+) plasmid of $E$. coli (strain K12). The GC content of the vaccine DNA sequence was $51.8 \%$ suggesting good expression of the vaccine in the $E$. coli host (Fig. 10):

TGCGCTGCTTACGTTCGTTTCGAAAACACCACCTCTGACAAAGGTAAAATCCCGTCTAAAGTTATCAAATCTTACTACGGTACCATGGACATCAAAAAAATCAACGAAGGT

\section{Discussion}

The catastrophic consequences of the ongoing COVID-19 pandemic have entailed a preemptive development of vaccines for all pandemic-prone diseases. NiV infection is a zoonotic disease that can be transmitted to humans directly from natural reservoirs or indirectly from other infected animals or through direct contact with other infected humans, underscoring the potential pandemic nature of the disease. Several approaches were used to develop vaccines against animal NiV infection, including the use of glycoprotein G, and protein F subunits as immunogens, non-replicating vectors, and DNA vaccine. These trials showed very promising outcomes. However, as yet, there are no approved vaccines against human infection, despite a number of ongoing clinical trials [5256]. In the present study, a multi-subunit vaccine was designed using the virus structural proteins, which are moderators of the infection process [2]. Unlike single viral protein-based vaccines, multi-epitope vaccines are believed to provide better immunity by inducing a broader immune response [57]. Furthermore, single viral protein-based vaccines are inclined to lose their efficiency due to the accumulation of mutations in the target protein, a phenomenon seldom noted in the multisubunit vaccine [58]. For designing the current candidate vaccine, initially, 6 NiV structural proteins were selected, however, upon primary screening for antigenicity, the large polymerase protein $(L)$ was excluded due to lack of antigenicity. Then the remaining 5 proteins were screened for potential epitopes, none of the CTL and HTL epitopes predicted from matrix protein (M) was eligible for selection in the final vaccine construct due to lack of antigenicity or toxicity. Proper presentation of these epitopes by the antigen-presenting cells (APC) and rapid and efficient immune response contingent upon accurate digestion of the constructed vaccine molecule by the proteasomes of different immune cells and binding transporters associated with antigen processing (TAP), therefore, the predicted epitopes were linked together using linkers that facilitate these cleavage and binding processes [59]. The addition of a cysteine residue to the N-terminal of the vaccine construct enhances the binding of the vaccine to the protein carrier, while the four amino acids EPEA were added to the C-terminal to facilitate the vaccine downstream purification [39]. The molecular weight of the candidate vaccine (54.6 kDa) falls well with the optimum molecular weight for vaccines (40-70 kDa) [60]. The physiochemical properties results showed a soluble, thermostable, hydrophilic construct, suggesting an adequate vaccine accessibility, absorption [61], and rapid sustainable immune response. The secondary structure of the protein molecule is a key determinant of its tertiary structure, the predicted structure revealed a predominant helix and coiled coil secondary structures.

The validation results of the 3D structure model of the vaccine molecule showed an acceptable model with $93.1 \%$ of the residues falling within the favorable region in Ramachandran plot and overall quality score of $90.4 \%$ in addition to a Z-score of -3.84 [62]. An appropriate immune response requires recognition 
and binding of the vaccine molecule by the toll-like receptors, therefore, such binding between the vaccine and the TLR-3 was studied through docking of the two molecules and the strength and stability of the interaction were assessed by molecular simulation and dynamics analysis, the estimated potential energy and RMSD plot showing RMSD value of $0.65 \mathrm{~nm}$ for the backbone of the docked complex, consequently, stable vaccine-TLR3 complex. The reverse vaccinology-multi-subunit vaccine designing is an alternative in silico approach used to overcome the downsides of the costly, time-consuming conventional vaccine development methods. This approach was previously employed for the development of multisubunit vaccines against a number of bacterial and viral diseases such as Streptococcus pneumoniae [63], SARS CoV2 [64], SARS [65], and Lassa virus [66].

Despite the promising results of the designed vaccine construct, the present study has nonetheless some limitations, including the need for experimental validation of the vaccine efficiency and further investigations on pathogenic priming and autoimmune induction are warranted.

\section{Conclusion}

The inclusion of the NiV infection in the WHO priority disease list for research and development in emergency contexts underlines the importance of rapid vaccine design and development for an emerging disease with epidemic potential. The current study is an attempt to develop a multisubunit vaccine effective enough to mount both humoral and cell medicated immune response to the viral structural proteins using reverse vaccinology tools.

In conclusion, the proposed vaccine fulfilled all antigenicity, immunogenicity, allergenicity, and physiochemical requirements of an efficacious multi-subunit vaccine against NiV infection in human.

\section{Abbreviations}




\begin{tabular}{|c|c|}
\hline ATP: & Transporter-associated antigen processing \\
\hline CD: & Cluster of differentiation \\
\hline COVID-19: & Coronavirus Disease of 2019 \\
\hline CTL: & Cytotoxic T lymphocyte \\
\hline DNA: & Deoxyribonucleic acid \\
\hline Fs: & Femtosecond \\
\hline GRAVY: & Grand average of hydropathicity \\
\hline GROMACS: & Groningen Machine for Chemical Simulation \\
\hline HeV: & Hendra virus \\
\hline HLA: & Human Leukocyte Antigen \\
\hline HTL: & Helper T lymphocyte \\
\hline IC: & Inhibitory Concentration \\
\hline IEDB: & Immune Epitope Database \\
\hline IFN-: & Interferon gamma \\
\hline IgG: & Immunoglobulin G \\
\hline Jcat: & JAVA codon adaptation tool \\
\hline K: & Kelvin \\
\hline $\mathrm{Kb}:$ & Kilo base \\
\hline Kcal: & Kilo calorie \\
\hline kDa: & Kilo Dalton \\
\hline MHC: & Major Histocompatibility Complex \\
\hline NCBI: & National center for Biotechnology Information \\
\hline NiV: & Nepha virus \\
\hline nM: & Nanometer \\
\hline NMR: & Nuclear Magnetic Resonance \\
\hline Ns: & Nanosecond \\
\hline PSSM: & Position specific scoring matrix \\
\hline RMSD: & Root Mean Square Deviation \\
\hline RNA: & Ribonucleic acid \\
\hline SARS: & Severe Acute Respiratory Syndrome \\
\hline SARS CoV2: & Severe Acute Respiratory Syndrome Coronavirus -2 \\
\hline SVM: & Support vector machine \\
\hline TLR: & Toll-like receptor \\
\hline VMD: & Molecular dynamics visualization \\
\hline WHO: & World Health Organization \\
\hline
\end{tabular}

\section{References}

1. Ambat AS, Zubair SM, Prasad N, Pundir P, Rajwar E, Patil DS, Mangad P. Nipah virus: A review on epidemiological characteristics and outbreaks to inform public health decision making. Journal of infection and public health. 2019 Sep 1;12(5):634-9.

2. Sun B, Jia L, Liang B, Chen Q, Liu D. Phylogeography, transmission, and viral proteins of Nipah virus. Virol Sin. 2018 Oct;33(5):385-93.

3. Ang BS, Lim TC, Wang L. Nipah virus infection. Journal of clinical microbiology. 2018 May 25;56(6):e01875-17.

4. Sendow I, Field HE, Adjid A, Ratnawati A, Breed AC, Morrissy C, Daniels P. Screening for Nipah virus infection in West Kalimantan province, Indonesia. Zoonoses Public Health. 2010 Dec;57(7-8):499-503.

5. Shariff M. Nipah virus infection: a review. Epidemiology \& Infection. 2019;147. 
6. Goh KJ, Tan CT, Chew NK, Tan PS, Kamarulzaman A, Sarji SA, Wong KT, Abdullah BJ, Chua KB, Lam SK. Clinical features of Nipah virus encephalitis among pig farmers in Malaysia. New England Journal of Medicine. 2000 Apr 27;342(17):1229-35.

7. Sejvar JJ, Hossain J, Saha SK, Gurley ES, Banu S, Hamadani JD, Faiz MA, Siddiqui FM, Mohammad QD, Mollah AH, Uddin R. Long-term neurological and functional outcome in Nipah virus infection. Annals of Neurology: Official Journal of the American Neurological Association the Child Neurology Society. 2007 Sep;62(3):235-42.

8. Wang LF, Harcourt BH, Yu M, Tamin A, Rota PA, Bellini WJ, Eaton BT. Molecular biology of Hendra and Nipah viruses. Microbes and infection. 2001 Apr 1;3(4):279- 87.

9. Marsh GA, De Jong C, Barr JA, Tachedjian M, Smith C, Middleton D, Yu M, Todd S, Foord AJ, Haring V, Payne J. Cedar virus: a novel Henipavirus isolated from Australian bats.

10. WHO. 2018. Nipah Virus Infection factsheet. http://www.who.int/ mediacentre/factsheets/fs262/en/ (accessed 07 June 2021$).$

11. Enchéry F, Horvat B. Understanding the interaction between henipaviruses and their natural host, fruit bats: Paving the way toward control of highly lethal infection in humans. International reviews of immunology. 2017 Mar 4;36(2):108-21.

12. Pelissier R, lampietro M, Horvat B. Recent advances in the understanding of Nipah virus immunopathogenesis and anti-viral approaches. F1000Research. $2019 ; 8$.

13. Pernet O, Wang YE, Lee B. Henipavirus receptor usage and tropism. Henipavirus. 2012:59-78.

14. Martinez-Gil L, Vera-Velasco NM, Mingarro I. Exploring the human-Nipah virus protein-protein interactome. Journal of virology. 2017 Nov 14;91(23):e01461-17.

15. Lee B, Ataman ZA. Modes of paramyxovirus fusion: a Henipavirus perspective. Trends in microbiology. 2011 Aug 1;19(8):389 - 99.

16. Weis M, Maisner A. Nipah virus fusion protein: importance of the cytoplasmic tail for endosomal trafficking and bioactivity. European journal of cell biology. 2015 Jul 1;94(7-9):316-22.

17. Sen N, Kanitkar TR, Roy AA, Soni N, Amritkar K, Supekar S, Nair S, Singh G, Madhusudhan MS. Predicting and designing therapeutics against the Nipah virus. PLoS neglected tropical diseases. 2019 Dec 12;13(12):e0007419.

18. Watkinson RE, Lee B. Nipah virus matrix protein: expert hacker of cellular machines. FEBS letters. 2016 Aug 1;590(15):2494 - 511.

19. Satterfield BA, Cross RW, Fenton KA, Borisevich V, Agans KN, Deer DJ, Graber J, Basler CF, Geisbert TW, Mire CE. Nipah virus C and W proteins contribute to respiratory disease in ferrets. Journal of virology. 2016 Jun 24;90(14):6326-43.

20. Mathieu C, Guillaume V, Volchkova VA, Pohl C, Jacquot F, Looi RY, Wong KT, Legras-Lachuer C, Volchkov VE, Lachuer J, Horvat B. Nonstructural Nipah virus $C$ protein regulates both the early host proinflammatory response and viral virulence. Journal of virology. 2012 Oct 1;86(19):10766-75.

21. Yoneda M, Georges-Courbot MC, Ikeda F, Ishii M, Nagata N, Jacquot F, Raoul H, Sato H, Kai C. Recombinant measles virus vaccine expressing the Nipah virus glycoprotein protects against lethal Nipah virus challenge. PloS one. 2013 Mar;14(3):e58414. 8(.

22. Griffin BD, Leung A, Chan M, Warner BM, Ranadheera C, Tierney K, Audet J, Frost KL, Safronetz D, Embury-Hyatt C, Booth SA. Establishment of an RNA polymerase II-driven reverse genetics system for Nipah virus strains from Malaysia and Bangladesh. Scientific reports. 2019 Aug 1;9(1):1-7.

23. Harcourt BH, Lowe L, Tamin A, Liu X, Bankamp B, Bowden N, Rollin PE, Comer JA, Ksiazek TG, Hossain MJ, Gurley ES. Genetic characterization of Nipah virus, Bangladesh, 2004. Emerging infectious diseases. 2005 Oct;11(10):1594.

24. Lo MK, Lowe L, Hummel KB, Sazzad HM, Gurley ES, Hossain MJ, Luby SP, Miller DM, Comer JA, Rollin PE, Bellini WJ. Characterization of Nipah virus from outbreaks in Bangladesh, 2008-2010. Emerging infectious diseases. 2012 Feb;18(2):248.

25. Doytchinova IA, Flower DR. VaxiJen: a server for prediction of protective antigens, tumour antigens and subunit vaccines. BMC Bioinform. 2007 Dec;8(1):1-7.

26. Larsen MV, Lundegaard C, Lamberth K, Buus S, Lund O, Nielsen M. Large-scale validation of methods for cytotoxic T-lymphocyte epitope prediction. BMC bioinformatics. 2007 Dec;8(1):1-2.

27. Gupta S, Kapoor P, Chaudhary K, Gautam A, Kumar R, Open Source Drug Discovery Consortium. Raghava GP. In silico approach for predicting toxicity of peptides and proteins. PloS one. 2013 Sep;13(9):e73957. 8(.

28. Calis JJ, Maybeno M, Greenbaum JA, Weiskopf D, De Silva AD, Sette A, Keşmir C, Peters B. Properties of MHC class I presented peptides that enhance immunogenicity. PLoS computational biology. 2013 Oct 24;9(10):e1003266.

29. Nielsen M, Lund O. NN-align. An artificial neural network-based alignment algorithm for MHC class II peptide binding prediction. BMC bioinformatics. 2009 Dec;10(1):1-0.

30. Saha S, Raghava GP. AlgPred: prediction of allergenic proteins and mapping of IgE epitopes. Nucleic acids research. 2006 Jul 1;34(suppl_2):W202-9.

31. Dhanda SK, Vir P, Raghava GP. Designing of interferon-gamma inducing MHC class-II binders. Biol Direct. 2013 Dec;8(1):1-5.

32. Bui HH, Sidney J, Dinh K, Southwood S, Newman MJ, Sette A. Predicting population coverage of T-cell epitope-based diagnostics and vaccines. BMC bioinformatics. 2006 Dec;7(1):1-5.

33. Yang Z, Bogdan P, Nazarian S. An in silico deep learning approach to multi-epitope vaccine design: a SARS-CoV-2 case study. Scientific reports. 2021 Feb;5(1):1-21. 11(

34. Larsen JE, Lund O, Nielsen M. Improved method for predicting linear B-cell epitopes. Immunome research. 2006 Dec;2(1):1-7.

35. Emini EA, Hughes JV, Perlow D, Boger J. Induction of hepatitis A virus-neutralizing antibody by a virus-specific synthetic peptide. Journal of virology. 1985 Sep;55(3):836-9. 
36. Kolaskar AS, Tongaonkar PC. A semi-empirical method for prediction of antigenic determinants on protein antigens. FEBS letters. 1990 Dec 10;276(12):172-4.

37. Gupta S, Ansari HR, Gautam A, Raghava GP. Identification of B-cell epitopes in an antigen for inducing specific class of antibodies. Biology direct. 2013 Dec;8(1):1-5.

38. Bandyopadhyay A, Cambray S, Gao J. Fast and selective labeling of N-terminal cysteines at neutral pH via thiazolidino boronate formation. Chemical science. 2016;7(7):4589-93.

39. Jin J, Hjerrild KA, Silk SE, Brown RE, Labbé GM, Marshall JM, Wright KE, Bezemer S, Clemmensen SB, Biswas S, Li Y. Accelerating the clinical development of protein-based vaccines for malaria by efficient purification using a four amino acid C-terminal 'C-tag'. International journal for parasitology. 2017 Jun $1 ; 47(7): 435-46$.

40. Pahari S, Chatterjee D, Negi S, Kaur J, Singh B, Agrewala JN. Morbid sequences suggest molecular mimicry between microbial peptides and self-antigens: a possibility of inciting autoimmunity. Frontiers in microbiology. 2017 Oct 9;8:1938.

41. Gasteiger E, Hoogland C, Gattiker A, Wilkins MR, Appel RD, Bairoch A. Protein identification and analysis tools on the ExPASy server. The proteomics protocols handbook. 2005:571-607.

42. Buchan DW, Jones DT. The PSIPRED protein analysis workbench: 20 years on. Nucleic acids research. 2019 Jul 2;47(W1):W402-7.

43. Sippl MJ. Recognition of errors in three-dimensional structures of proteins. Proteins: Structure, Function, and Bioinformatics. 1993 Dec;17(4):355-62.

44. Williams CJ, Headd JJ, Moriarty NW, Prisant MG, Videau LL, Deis LN, Verma V, Keedy DA, Hintze BJ, Chen VB, Jain S. MolProbity: More and better reference data for improved all-atom structure validation. Protein Sci. 2018 Jan;27(1):293-315.

45. Colovos C, Yeates TO. Verification of protein structures: patterns of nonbonded atomic interactions. Protein science. 1993 Sep;2(9):1511-9.

46. Schneidman-Duhovny D, Inbar Y, Nussinov R, Wolfson HJ. PatchDock and SymmDock: servers for rigid and symmetric docking. Nucleic acids research. 2005 Jul 1;33(suppl_2):W363-7.

47. Honorato RV, Koukos PI, Jiménez-García B, Tsaregorodtsev A, Verlato M, Giachetti A, Rosato A, Bonvin AM. Structural biology in the clouds: The WeNMREOSC Ecosystem. Frontiers in Molecular Biosciences. 2021:708.

48. Sastry GM, Adzhigirey M, Day T, Annabhimoju R, Sherman W. Protein and ligand preparation: parameters, protocols, and influence on virtual screening enrichments. J Comput Aided Mol Des. 2013 Mar;27(3):221-34.

49. Rapin N, Lund O, Bernaschi M, Castiglione F. Computational immunology meets bioinformatics: the use of prediction tools for molecular binding in the simulation of the immune system. PloS one. 2010 Apr 16;5(4):e9862.

50. Grote A, Hiller K, Scheer M, Münch R, Nörtemann B, Hempel DC, Jahn D. JCat: a novel tool to adapt codon usage of a target gene to its potential expression host. Nucleic acids research. 2005 Jul 1;33(suppl_2):W526-31.

51. Ali M, Pandey RK, Khatoon N, Narula A, Mishra A, Prajapati VK. Exploring dengue genome to construct a multi-epitope based subunit vaccine by utilizing immunoinformatics approach to battle against dengue infection. Scientific reports. 2017 Aug 23;7(1):1-3.

52. Broder CC, Weir DL, Reid PA. Hendra virus and Nipah virus animal vaccines. Vaccine. 2016 Jun 24;34(30):3525-34.

53. Wang X, Ge J, Hu S, Wang Q, Wen Z, Chen H, Bu Z. Efficacy of DNA immunization with F and G protein genes of Nipah virus. Annals of the New York Academy of Sciences. 2006 Oct;1081(1):pp. 243-5.

54. Prescott J, de Wit E, Feldmann H, Munster VJ. The immune response to Nipah virus infection. Archives of virology. 2012 Sep;157(9):1635-41.

55. Guillaume V, Contamin H, Loth P, Georges-Courbot MC, Lefeuvre A, Marianneau P, Chua KB, Lam SK, Buckland R, Deubel V, Wild TF. Nipah virus: vaccination and passive protection studies in a hamster model. Journal of virology. 2004 Jan;15(2):834-40. 78(.

56. Broder CC. Henipavirus outbreaks to antivirals: the current status of potential therapeutics. Current opinion in virology. 2012 Apr 1;2(2):176 - 87.

57. Saadi M, Karkhah A, Nouri HR. Development of a multi-epitope peptide vaccine inducing robust $T$ cell responses against brucellosis using immunoinformatics based approaches. Infection Genetics Evolution. 2017 Jul;1:51:227-34.

58. Keshwara R, Shiels T, Postnikova E, Kurup D, Wirblich C, Johnson RF, Schnell MJ. Rabies-based vaccine induces potent immune responses against Nipah virus. npj Vaccines. 2019 Apr 15;4(1):1-0.

59. Nezafat N, Ghasemi Y, Javadi G, Khoshnoud MJ, Omidinia E. A novel multi-epitope peptide vaccine against cancer: an in silico approach. Journal of theoretical biology. 2014 May 21;349:121 - 34.

60. Nezafat N, Ghasemi Y, Javadi G, Khoshnoud MJ, Omidinia E. A novel multi-epitope peptide vaccine against cancer: an in silico approach. Journal of theoretical biology. 2014 May 21;349:121 - 34.

61. Savjani KT, Gajjar AK, Savjani JK. Drug solubility: importance and enhancement techniques. International Scholarly Research Notices. $2012 ; 2012$.

62. Kunz S. Receptor binding and cell entry of Old World arenaviruses reveal novel aspects of virus-host interaction. Virology. 2009 May 10;387(2):245-9.

63. Dorosti H, Eslami M, Negahdaripour M, Ghoshoon MB, Gholami A, Heidari R, Dehshahri A, Erfani N, Nezafat N, Ghasemi Y. Vaccinomics approach for developing multi-epitope peptide pneumococcal vaccine. Journal of Biomolecular Structure and Dynamics. 2019 Jan 11.

64. Adam KM. Immunoinformatics approach for multi-epitope vaccine design against structural proteins and ORF1a polyprotein of severe acute respiratory syndrome coronavirus-2 (SARS-CoV-2). Tropical Diseases, Travel Medicine and Vaccines. 2021 Dec;7(1):1-3.

65. Srivastava S, Kamthania M, Kumar Pandey R, Kumar Saxena A, Saxena V, Kumar Singh S, Kumar Sharma R, Sharma N. Design of novel multi-epitope vaccines against severe acute respiratory syndrome validated through multistage molecular interaction and dynamics. Journal of Biomolecular Structure and Dynamics. 2019 Nov 2;37(16):4345-60. 
66. Omoniyi AA, Adebisi SS, Musa SA, Nzalak JO, Danborno B, Bauchi ZM, Badmus IT, Olatomide OD, Oladimeji OJ, Nyengaard JR. Designing a multi-epitope vaccine against the Lassa virus through reverse vaccinology, subtractive proteomics, and immunoinformatics approaches. Informatics in Medicine Unlocked. 2021 Jan 1;25:100683.

\section{Declarations}

Acknowledgement: The author is indebted to the faculty members of college of applied medical sciences and the deanship of scientific research, university of Bisha, for their help and support

Funding: This research did not receive any specific grant from funding agencies in the public, commercial, or not-for-profit sectors.

Competing interests: The author declares that he has no competing interests

Availability of data: All data supporting the conclusions are included in the main manuscript

Consent for publication: Author has agreed for manuscript publication

Ethics approval: No applicable

Author's contributions: The manuscript was entirely prepared by a single author

\section{Figures}

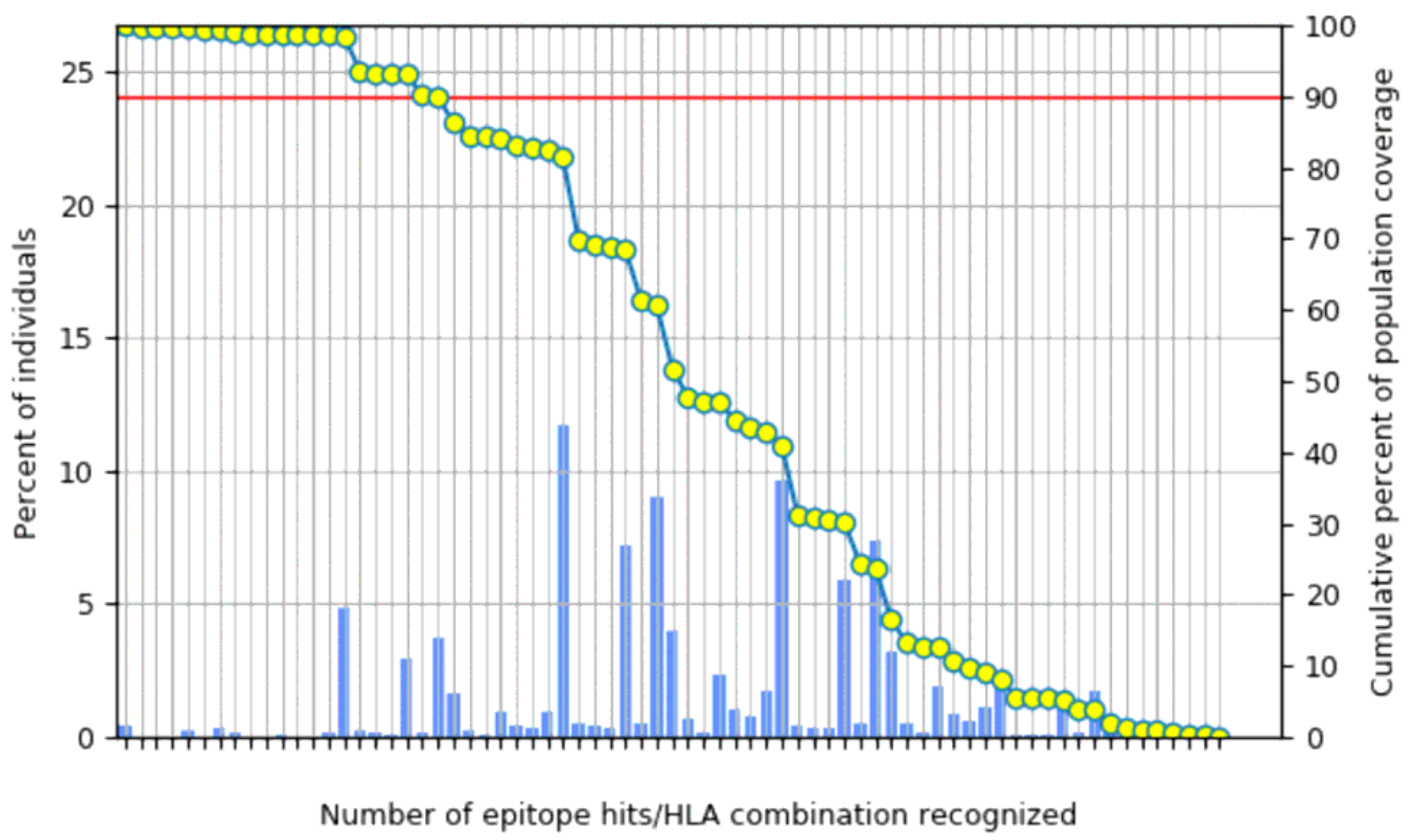

Figure 1

The coverage of HLA class I restricted epitopes was $98.59 \%$ of the world population, and the coverage of HLA class II restricted epitopes was $69.81 \%$ of the world population, while the coverage of the combined HLA class I and class II was $99.57 \%$ of the world population, this suggests a remarkably high population coverage of the given multisubunit vaccine. 


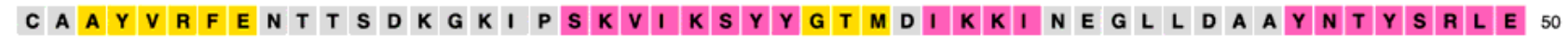

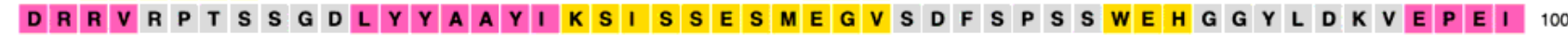

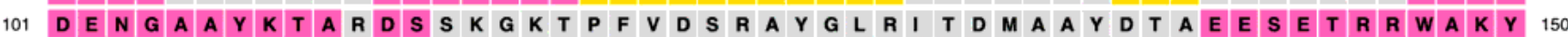

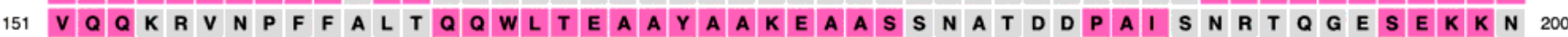

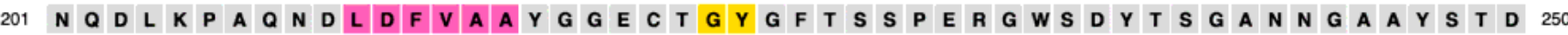

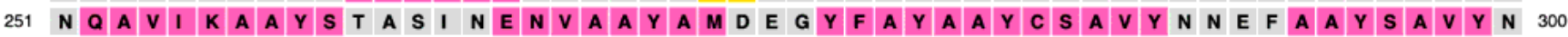

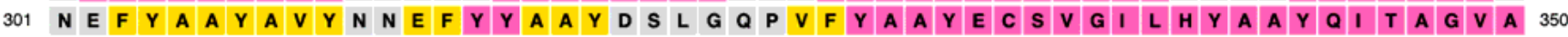

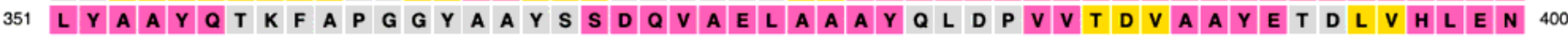

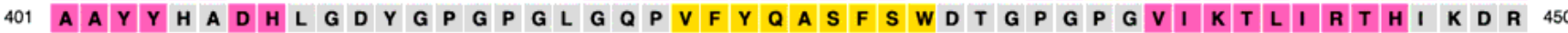

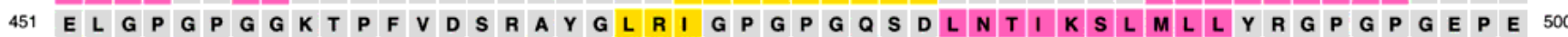
501 A

10 20 30 40

\begin{tabular}{|c|c|c|c|}
\hline Strand & Helix & Coil & Disordered \\
\hline Disordered, protein binding & Putative Domain Boundary & Membrane Interaction & Transmembrane Helix \\
\hline Extracellular & Re-entrant Helix & Cytoplasmic & Signal Peptide \\
\hline
\end{tabular}

Figure 2

The results of secondary structure prediction by PSIPRED sever showed predominant helix and coil structures.
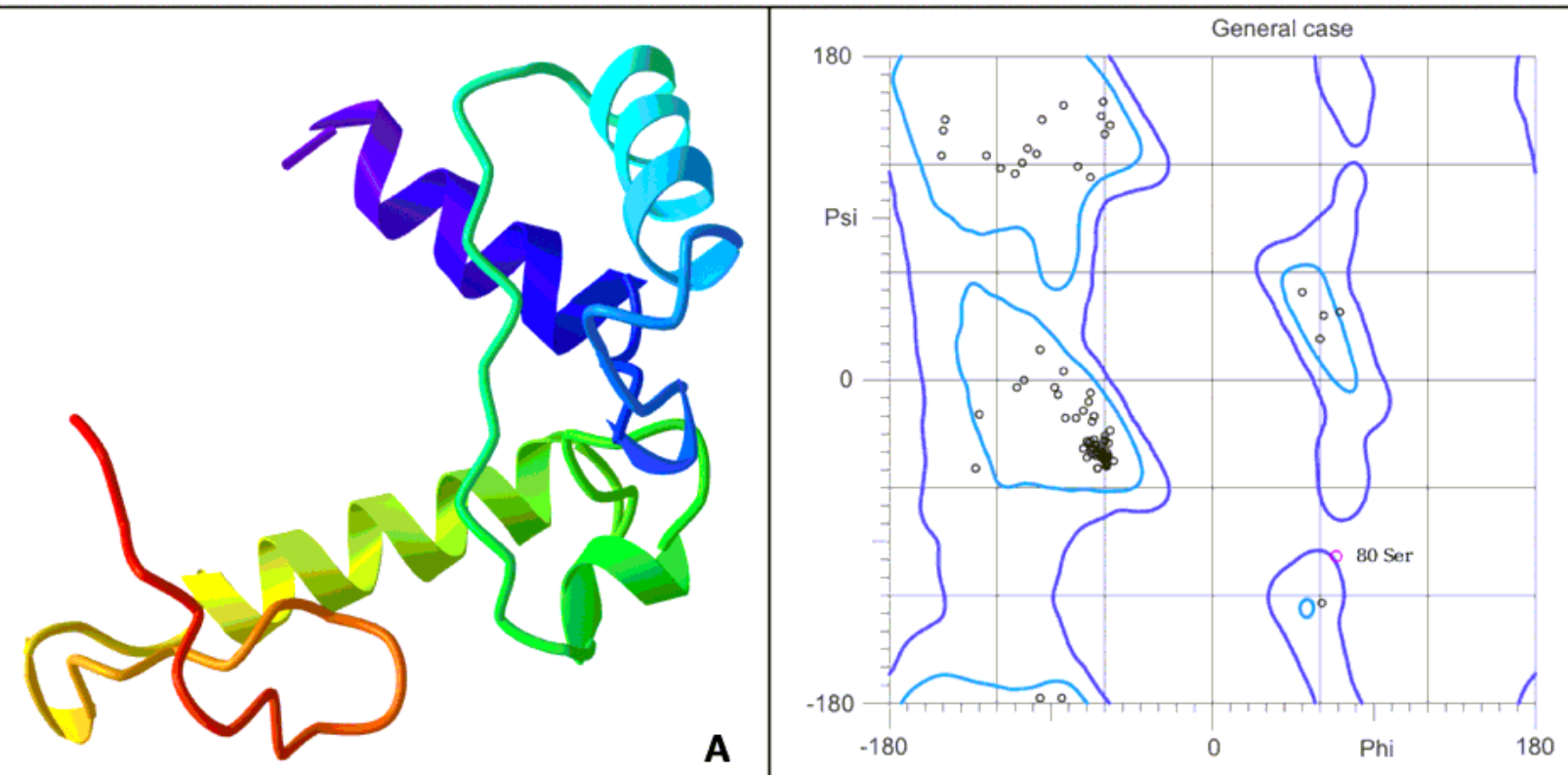

B

Figure 3

The best-predicted model showed $93.1 \%$ of the residues fall within the favorable region in the Ramachandran plot. 


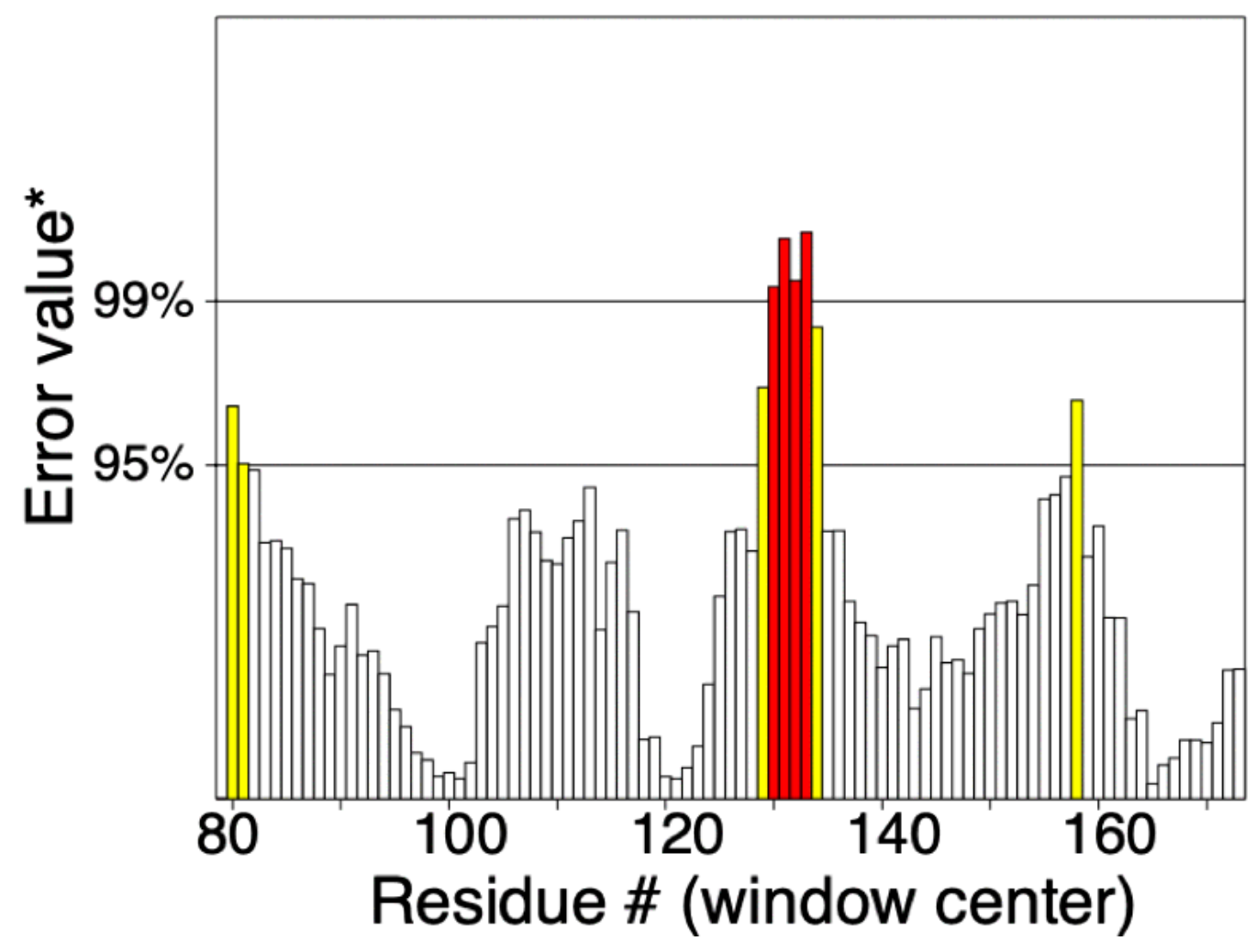

Figure 4

The value is less than the $91 \%$ threshold for an average quality.

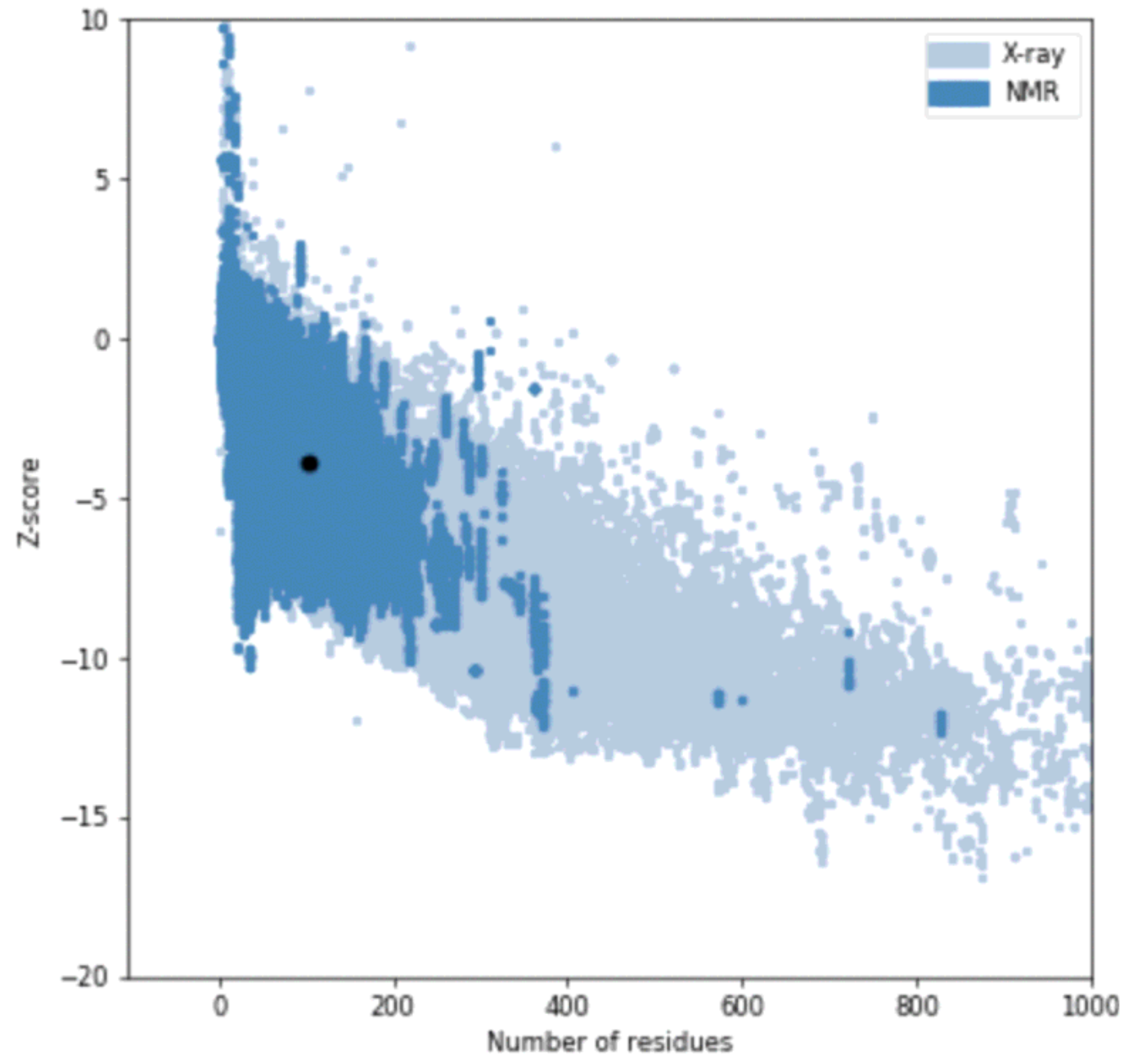

Page 13/18 
Figure 5

The model also showed a Z-score of -3.84 determined by both $\mathrm{x}$-ray crystallography and NMR analysis.

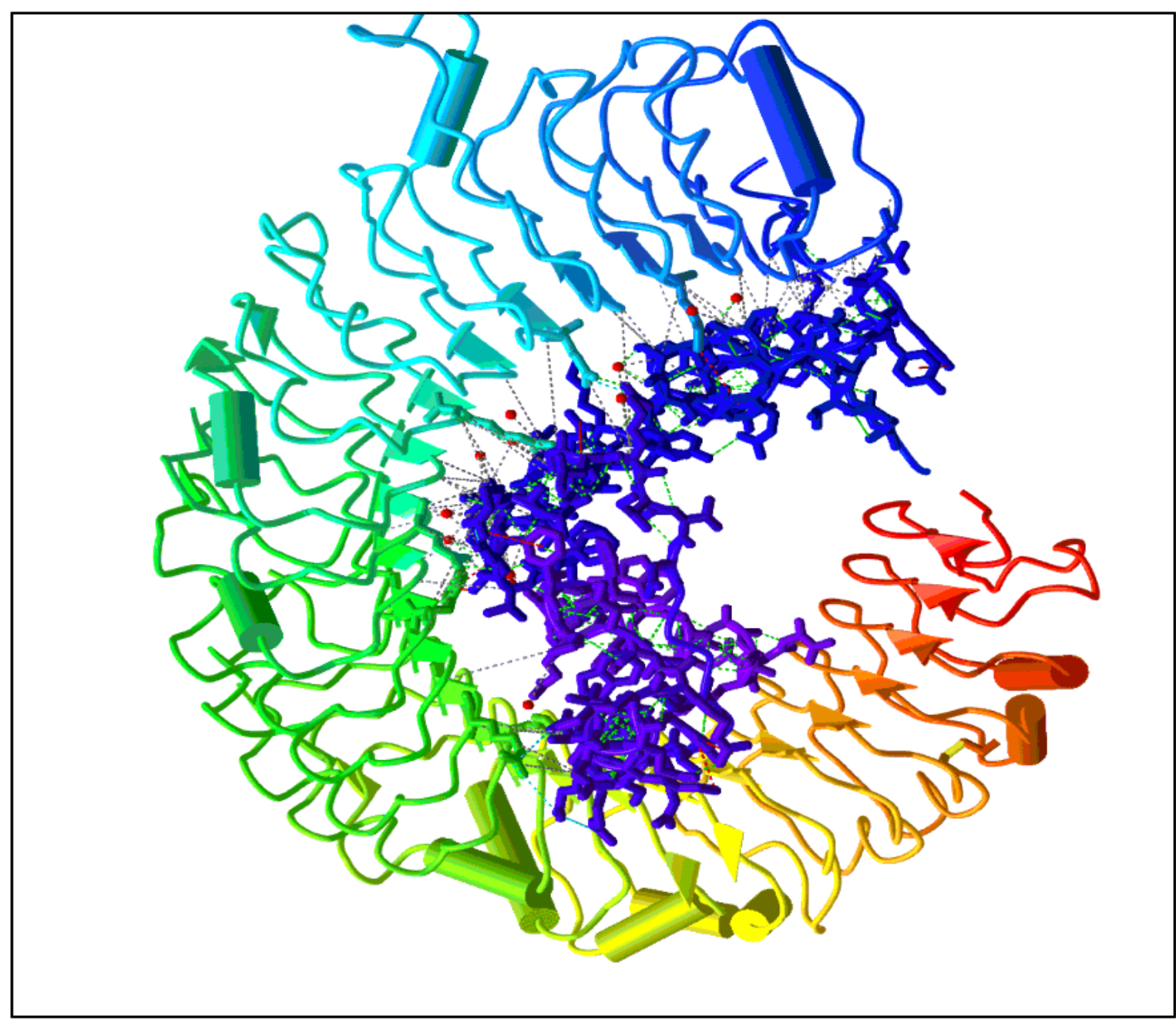

Figure 6

The selected docking solution had a globale energy of $-1.58 \mathrm{Kcal} / \mathrm{mol}$, attractive van der Waals force of $-9.13 \mathrm{Kcal} / \mathrm{mol}$, repulsive van der Waals force of 1.83 , $\mathrm{Kcal} / \mathrm{mol}$, and atomic contact energy of $2.98 \mathrm{Kcal} / \mathrm{mol}$. 


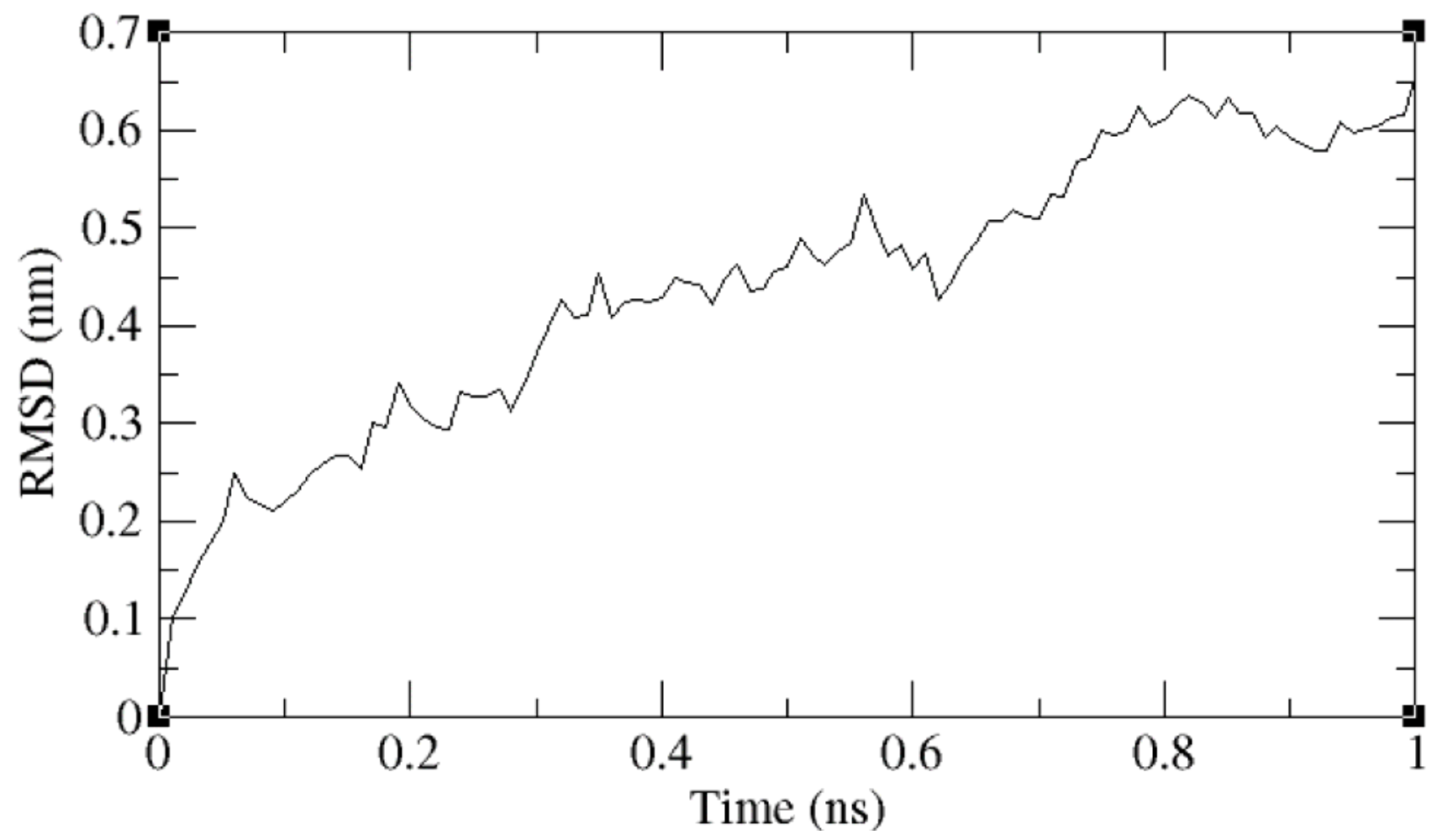

Figure 7

The molecular dynamics simulation results showed a root mean square deviation (RMSD) of $0.65 \mathrm{~nm}$ and the radius of gyration value with a relatively minor fluctuation throughout the 1000 ps period of simulation. 


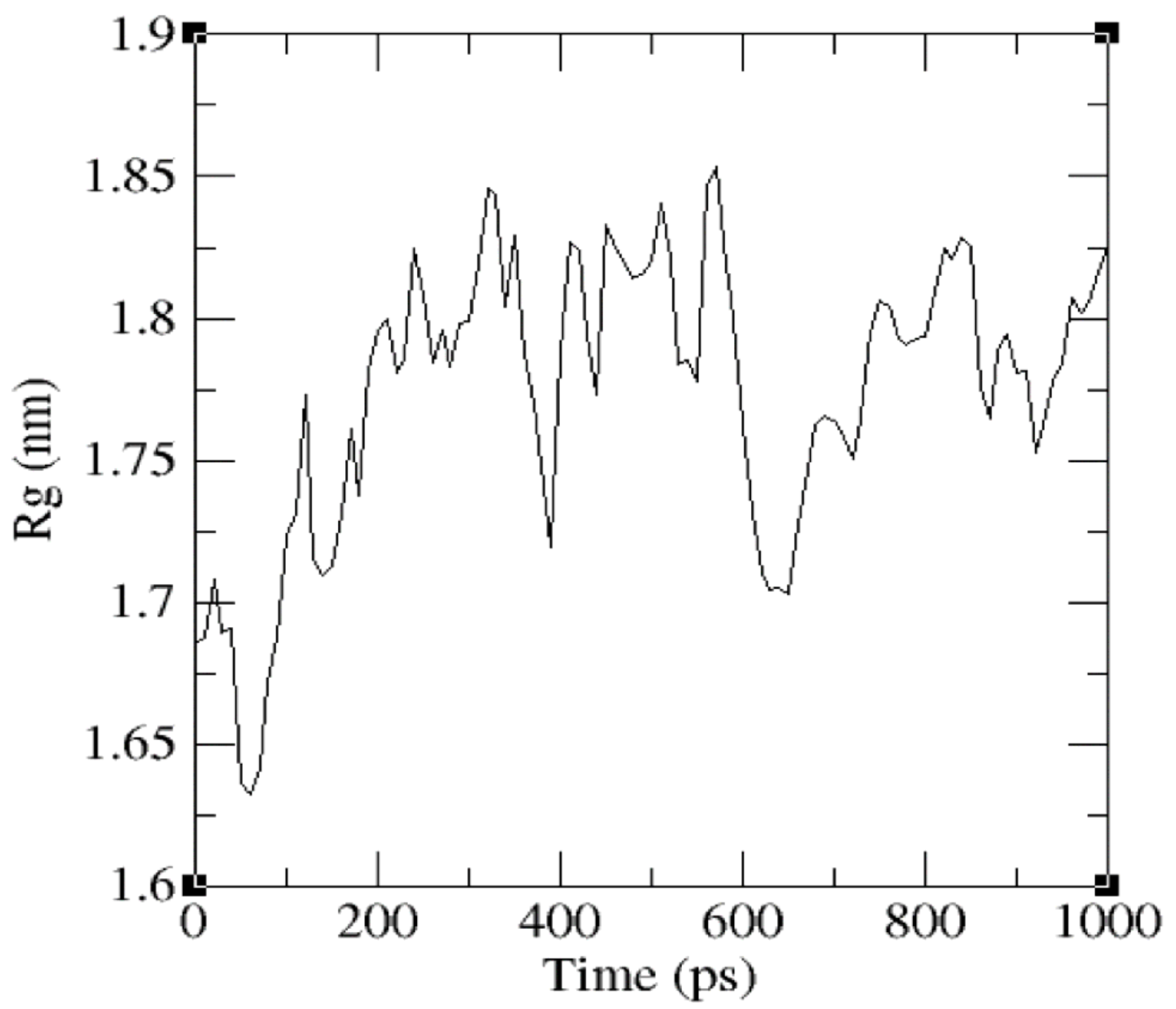

Figure 8

The molecular dynamics simulation results showed a root mean square deviation (RMSD) of $0.65 \mathrm{~nm}$ and the radius of gyration value with a relatively minor fluctuation throughout the 1000 ps period of simulation. 


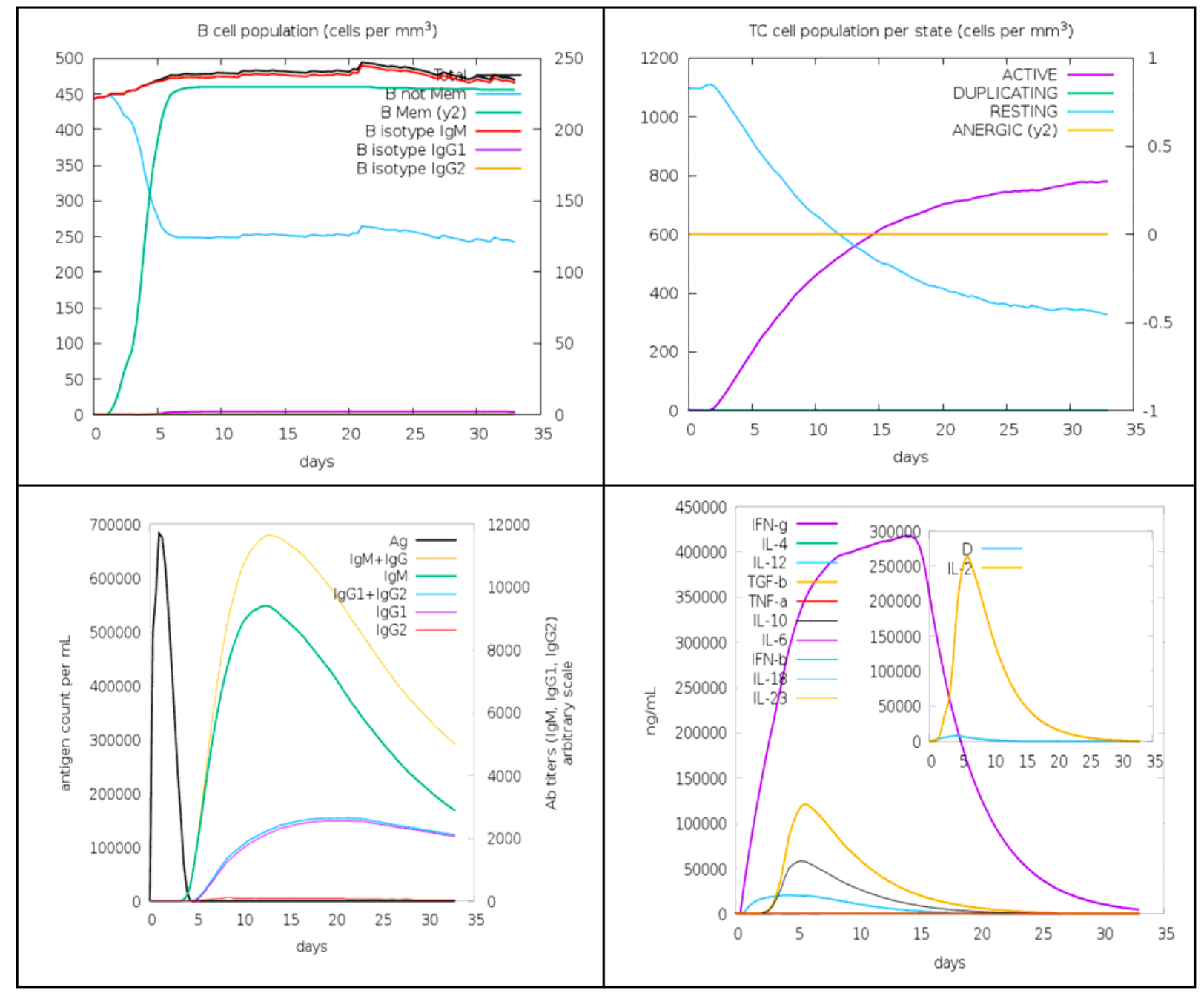

Figure 9

The concentration of cytokines and interleukins showed for more than half the simulation period followed by a gradual decline. 


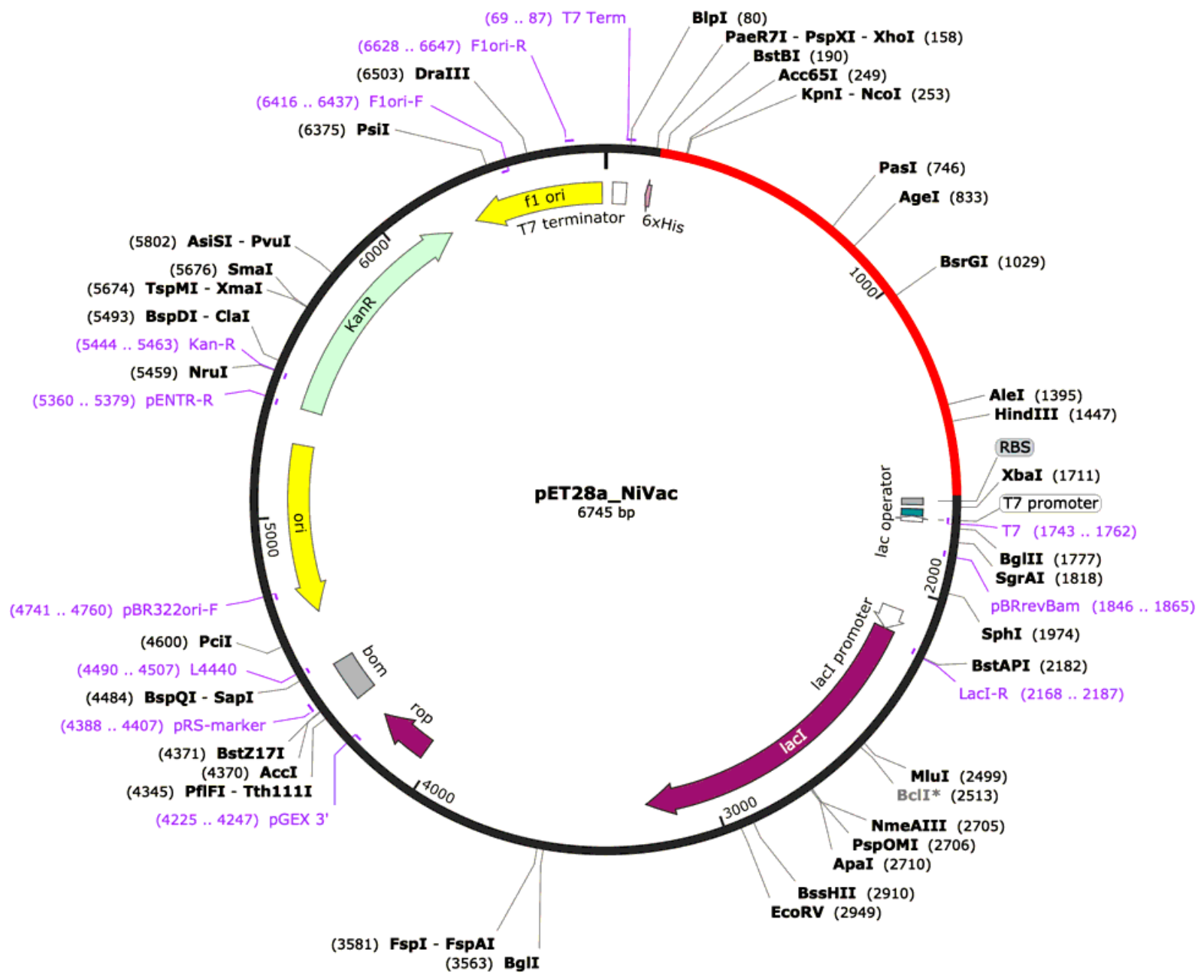

Figure 10

The GC content of the vaccine DNA sequence was $51.8 \%$ suggesting good expression of the vaccine in the E. coli host. 